\title{
Contrast and frequency competition for orientation-contingent color aftereffects
}

\author{
MARTY J. SCHMIDT \\ University of New Hampshire, Durham, New Hampshire 03824 \\ and \\ RONALD A. FINKE \\ Massachusetts Institute of Technology, Cambridge, Massachusetts 02139
}

\begin{abstract}
A "competition" paradigm was developed to examine separately the effects of pattern contrast and spatial frequency characteristics on the strength of orientation-contingent color aftereffects (McCollough effects). After adapting to alternately presented red/black and green/ black square-wave gratings (one horizontal, one vertical), 11 subjects viewed seven different kinds of test patterns. Unlike standard McCollough effect test stimuli, the present patterns had variable luminance profiles running both horizontally and vertically within each test pattern area. Forced choice responses were used to determine which aftereffect color (red or green) appeared, as characteristics of vertical and horizontal luminance profiles were varied separately among test stimulus types. We conclude that pattern contrast and human contrast sensitivity account for aftereffect colors in such stimuli. When contrast is taken into consideration, aftereffects are not predicted by similarity between adaptation and test pattern Fourier characteristics, nor are they predicted by the width, per se, of pattern elements.
\end{abstract}

McCollough (1965) first demonstrated patterncontingent color aftereffects by adapting subjects to orange and black vertical gratings alternated with blue and black horizontal gratings. The remarkable aspect of this effect (known generally as the McCollough effect) was that aftereffect colors were contingent on the orientation of achromatic test gratings. Vertical and horizontal test gratings were seen tinged with complementaries of their adapting colors. Other investigators have since produced some interesting accounts of the effect's dynamic properties, and similar color aftereffects contingent on pattern variables other than orientation have also been demonstrated (see Skowbo, Timney, Gentry, \& Morant, 1975).

Despite the wealth of McCollough effect data available, we cannot state with certainty which stimulus characteristics are actually necessary to establish and elicit pattern-contingent color aftereffects. Most McCollough effect demonstrations identify sufficient rather than necessary conditions for obtaining the effect. A major problem in this regard is that McCollough effect reports usually describe situations in which several stimulus characteristics vary together. It is often unclear which pattern variables are critical

The authors wish to thank Adam Reeves and C. R. Cavonius for helpful criticisms. Marty J. Schmidt's present address is: Institut für Arbeitsphysiologie an der Universität Dortmund, Postfach 1508 , D-4600 Dortmund 1, West Germany. Requests for reprints should be sent to Ronald A. Finke, Department of Psychology, E10-114A, Massachusetts Institute of Technology, Cambridge, Massachusetts 02139. for obtaining the aftereffects, and which variables simply covary with the critical variables. Our intent in the present study was to create a situation in which the effects of several test pattern characteristics could be examined separately. Specifically, we wanted to determine whether orientation-contingent color aftereffects are more effectively controlled by test pattern contrast characteristics or by pattern Fourier characteristics. We also examined the possibility that the width of black pattern elements is a critical factor in eliciting the effect.

\section{THE COMPETITION PARADIGM}

The present investigation was conducted using a competition paradigm that may be explained through reference to Figure 1. The figure shows two gratings viewed alternately during the adaptation phase of experimental sessions. After alternately viewing these particular gratings, we expect vertical test gratings to elicit a red aftereffect and horizontal gratings to elicit a green aftereffect: these results would correspond to a "standard" McCollough effect.

Instead of viewing gratings, however, our subjects viewed black and white test patterns of the type shown schematically at the bottom of Figure 1. Each half of the test pattern contained small black elements arranged so as to form large bars of the same size as adaptation grating bars. Notice that in each half of the test pattern, small elements had edges perpendicular to the edges of the larger bars they formed. Thus, 
each half of the test pattern contained vertical and horizontal edges. Unlike standard McCollough effect test stimuli, each half of the test pattern had a variable luminance profile in two orientations, rather than one. By changing the size and spacing of small elements among test stimuli (as explained below), we varied the characteristics of horizontal and vertical luminance profiles separately. This allowed us to arrange for "competition" within the same test area between a horizontal luminance profile having a specific contrast and a vertical profile having another specific contrast. Similarly, we could place a profile having one set of Fourier characteristics in competition with a perpendicular profile having a different set of Fourier characteristics. Our method, essentially, was to observe which aftereffect (red or green) appeared with such competitions. For example, if the aftereffect presumably associated with vertical gratings appeared, we assumed that the profile defining a vertical pattern produced a stronger aftereffect than the profile defining a horizontal pattern. By observing the outcomes of a number of these competitions, between selected contrast values and between selected sets of Fourier characteristics, we hoped to assess separately the effects of these pattern characteristics on aftereffect strength.

\section{METHOD}

\section{Subjects}

Eleven graduate students at the University of New Hampshire participated as subjects. All produced normal color vision scores on the Dvorine Color Vision Test and acuity ratings of $20 / 20$ with a standard Snellen chart (some were corrected to 20/20 with spectacles or contact lenses). Only when informed at the conclusion of the experiment were these subjects aware of the purpose of the study.

\section{Stimuli}

Each of the two adaptation patterns consisted of 10 cycles of a 2 cycles/deg (cpd) square-wave grating, and filled a square area $5^{\circ} \times 5^{\circ}$. Five subjects adapted to the red-horizontal green-vertical combinations shown in Figure 1; the six other subjects adapted to the reverse color-orientation combinations.

Stimuli were prepared as slides and projected onto a screen $4.5 \mathrm{~m}$ in front of the subject. Colored bars were produced by superimposing Kodak Wratten gelatin filters and achromatic transparencies of the bar patterns. The filters had the following dominant wavelengths under the tungsten illuminant used: red (Wratten No. 25) $617 \mathrm{~nm}$; green (Wratten No. 57A) $521 \mathrm{~nm}$. Neutral density filters brought luminances of colored and black bars to 120 and $5 \mathrm{~cd} / \mathrm{m}^{2}$, respectively, as measured with a Macbeth illuminometer.

Test stimuli, shown schematically in Figure 1, were achromatic and also filled a $5^{\circ} \times 5^{\circ}$ square area. The width and spacing of small black elements were varied among test stimuli so as to vary contrast and Fourier characteristics along horizontal and vertical dimensions.

Figure 2 illustrates our method for describing stimulus characteristics. The upper part of this figure shows an enlarged portion of the left half of the test pattern in Figure 1. One axis (vertical in the example) was perpendicular to the large bars. We call this the $y$-axis in all test stimuli, whether it ran vertically or horizontally. As shown in Figure 1, the y-axis in one half of the

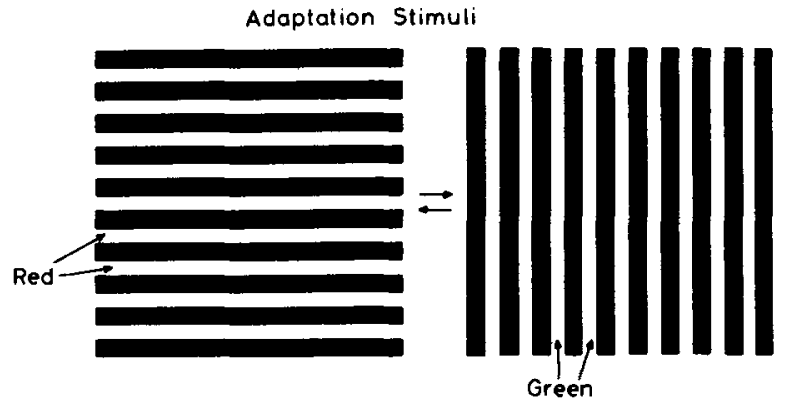

Sample Test Stimulus

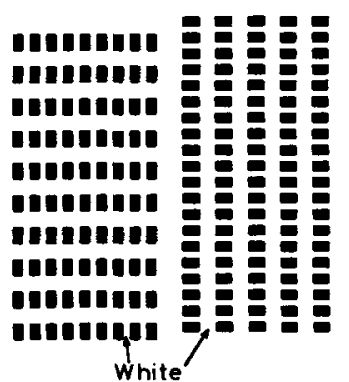

Figure 1. Schematic representation of pattern stimuli. The two alternately viewed colored adaptation gratings consisted of 10 cycles of a 2-cpd square-wave pattern and filled a $5^{\circ} \times 5^{\circ}$ square. Achromatic test patterns also filled a $5^{\circ} \times 5^{\circ}$ square. Two-cycles/deg bars (formed by rows or columns of small black elements) in one half of the test pattern were perpendicular to the larger bars they formed, creating the "competition" situation described in the text. If, for example, a red aftereffect appeared in the right half of this test pattern, we assumed that the luminance profile taken across the larger bars produced a stronger McCollough effect than the luminance profile taken across the small elements (see text for further explanation).

test stimulus was perpendicular to the $y$-axis in the other half of the test stimulus. The luminance distribution, when collapsed onto this axis, always had a square-wave profile with a spatial period $(\mathrm{Y})$ of $.5^{\circ}$. Thus, test pattern $y$-axis profiles were identical in size to adaptation pattern profiles. The other axis, which ran perpendicular to the small black pattern elements, was called the $\mathrm{x}$-axis.

The lower half of Figure 2 shows the luminance profile collapsed separately across the $\mathrm{x}$ and $\mathrm{y}$ axes. $\mathrm{L}_{\mathrm{o}}$ was the luminance in homogeneous black areas $\left(5 \mathrm{~cd} / \mathrm{m}^{2}\right)$ and $L_{\max }$ was the luminance in homogeneous white areas $\left(120 \mathrm{~cd} / \mathrm{m}^{2}\right)$. In deriving test pattern characteristics, we treated these profiles as infinite waveforms. ${ }^{1}$ The $\mathrm{x}$-axis spatial period was designated $\mathrm{X}$ and the $\mathrm{y}$-axis period was designated $Y$.

We varied contrast in two dimensions by varying the duty cycle of the x-axis luminance profile. As shown in Figure 2, we defined the duty cycle $\beta$ in either dimension as the size of one cycle's $L_{\text {max }}$ portion ( $x$ or $y$ ) divided by the period ( $\mathrm{X}$ or $\mathrm{Y}$ ). $\beta_{\mathrm{x}}$ was thus $\mathrm{X} / \mathrm{X}$ and $\beta_{\mathrm{y}}$ was $\mathrm{y} / \mathrm{Y}$. $\beta_{\mathrm{y}}$ was .5 for all stimuli, so that $\mathrm{y}$-axis profiles were always square waves.

Calculations of contrast for the $x$ and $y$ components of the patterns treated each axis as though it contained a grating of stripes. Consider as an example the right half of the sample test stimulus shown in Figure 1. Bright "stripes" of the vertical "grating" have a luminance throughout of $L_{\max }$. The dark "stripes" have an effective overall luminance of $\beta_{x} L_{\text {max }}+(1-$ $\left.\beta_{x}\right) L_{o}$; we call this dark stripe luminance $L_{y-\min }$ ' when referring to the stripes defined by the y-axis. Since the bright and dark 

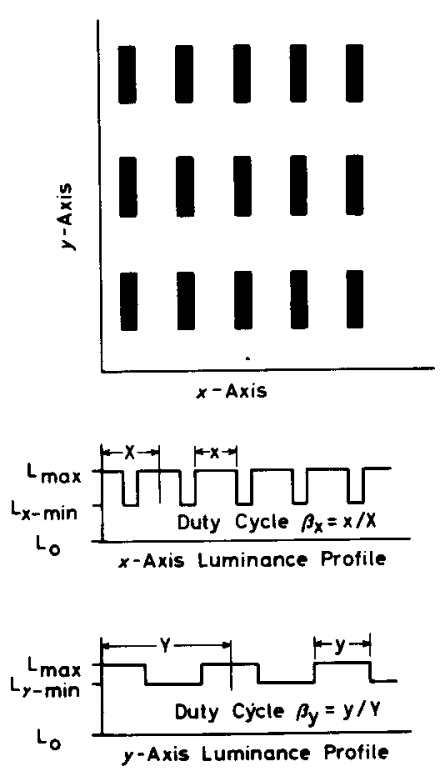

Figure 2. An enlargement of a small section of the left half of the test pattern from Figure 1 (top), and luminance profiles for this test pattern half taken horizontally (middle) and vertically (bottom). One of these profiles was identical in size to the luminance profile across adaptation bars (not graphed). This was designated the $y$-axis profile, whether it appeared vertically or horizontally; the perpendicular profile was designated the $x$-axis profile. Note that the $y$-axis profile in one test pattern half was perpendicular to the $y$-axis profile in the other test pattern half. Within each test pattern half, the $y$-axis profile was perpendicular to the $\mathrm{x}$-axis profile. $A$ horizontal profile defined a vertical pattern and a vertical profile defined a horizontal pattern. Thus, the two perpendicular profiles were competing for different color aftereffects. The lower half of this figure shows how the duty cycle $\beta$ for each profile was defined. $L_{\max }$ and $L_{0}$ were 120 and $5 \mathrm{~cd} / \mathrm{m}^{2}$, respectively. Computation of $L_{y-\min }$ and $L_{x-\min }$ is described in the text.

vertical "stripes" have equal width, the space-average luminance (I) is:

$$
\overline{\mathrm{L}}=.5\left[\mathrm{~L}_{\max }+\beta_{\mathrm{x}} \mathrm{L}_{\max }+\left(1-\beta_{\mathrm{x}}\right) \mathrm{L}_{\mathrm{o}}\right]
$$

The contrast of this vertical "grating," that is, contrast of the y-axis profile, is defined here (after Campbell \& Robson, 1968; see Footnote 2) as:

$$
\mathrm{m}_{y}=\frac{\mathrm{L}_{\max }-\mathrm{L}_{\mathrm{y}-\min }}{2 \overline{\mathrm{L}}}=\frac{\mathrm{L}_{\max }-\left[\beta_{\mathrm{x}} \mathrm{L}_{\max }+\left(1-\beta_{\mathrm{x}}\right) \mathrm{L}_{\mathrm{o}}\right]}{\mathrm{L}_{\max }+\beta_{\mathrm{x}} \mathrm{L}_{\max }+\left(1-\beta_{\mathrm{x}}\right) \mathrm{L}_{\mathrm{o}}} .
$$

On the same test pattern half, consider now the horizontal "grating" as the x-axis component. Bright "stripes" of relative width $\beta_{x}$ are illuminated by $L_{\text {max }}$, while dark "stripes" of relative width $\left(1-\beta_{x}\right)$ have an effective luminance of $.5 L_{\max }+.5 L_{o}$. Contrast of the horizontal pattern component, $\mathrm{m}_{\mathrm{x}}$, was calculated through appropriate substitutions of $\mathrm{L}_{x-\min }$ for $\mathrm{L}_{\mathrm{y}-\min }$ in Equation 2.

Seven different types of test stimuli were used, differing in $\mathrm{x}$-axis duty cycle and in size of spatial period $\mathrm{X}$; these are illustrated in Figure 3. The upper panel of Figure 4 shows how contrast of the test patterns in both dimensions varied with the value of $\beta_{x}$. Here, the smooth curves show values of $m_{y}$ and $m_{x}$ that would occur (with the present $L_{\max }, L_{0}$, and $\beta_{y}$ ) if $\beta_{x}$ were varied continuously from .01 to .99 . Letters and vertical lines locate test stimuli actually used in the experiment. Note that stimuli were included having $m_{x}>m_{y}$ (types $A$ and $B$ ), $m_{x}=m_{y}$ (types $C$, $D$, and $G$ ), and $m_{x}<m_{y}$ (types $E$ and $F$ ).

It should be noted that the average luminance $\overline{\mathrm{L}}$ of the test patterns varied in a way that was generally confounded with the contrast variable. However, $\bar{L}$ changed by less than a factor of 2 among patterns, and, in our analysis of results, the critical comparisons are among three patterns with the same $\bar{L}$.

In deriving Fourier characteristics of these stimuli, the adaptation pattern peak-to-peak luminance difference $\left(\mathrm{L}_{\max }-\mathrm{L}_{\mathrm{o}}=\right.$ $115 \mathrm{~cd} / \mathrm{m}^{2}$ ) was arbitrarily taken to be an amplitude of 1.0 . Using this convention, and treating luminance profiles as even functions of infinite extent, each profile can be synthesized from a series of harmonically related cosinusoidal waveforms; along the $x$-axis, amplitude $A_{n}$ of the $n^{\text {th }}$ harmonic component was specified as

$$
A_{n}=\left[\frac{L_{\max }-L_{x-\min }}{L_{\max }-L_{o}}\right] \frac{2}{n \pi} \sin n \beta_{x} \pi
$$

Amplitudes in the $y$ dimension were obtained by substituting $L_{y-\min }$ for $L_{x-\min }$ and $\beta_{y}$ for $\beta_{x}$ in Equation 3 above.

Figure 5 shows the frequency spectra in $x$ and $y$ dimensions' for our test stimuli. We emphasize two characteristics of these spectra: (1) y-axis profiles were the same size as adaptation pattern profiles; thus, all adaptation pattern profiles and test-pattern y-axis profiles had Fourier components at the same spatial frequencies. The fundamental component appeared at $2 \mathrm{cpd}$; progressively lower amplitude components appeared at higher odd harmonics (a 3rd harmonic at $6 \mathrm{cpd}$, a 5 th harmonic at $10 \mathrm{cpd}$, and so on). (2) Adaptation pattern profiles (not graphed) had no "x-axis" variation and therefore no $x$-axis Fourier components. Each test pattern type, however, had a different set of $\mathrm{x}$-axis Fourier components, as shown in Figure 5.

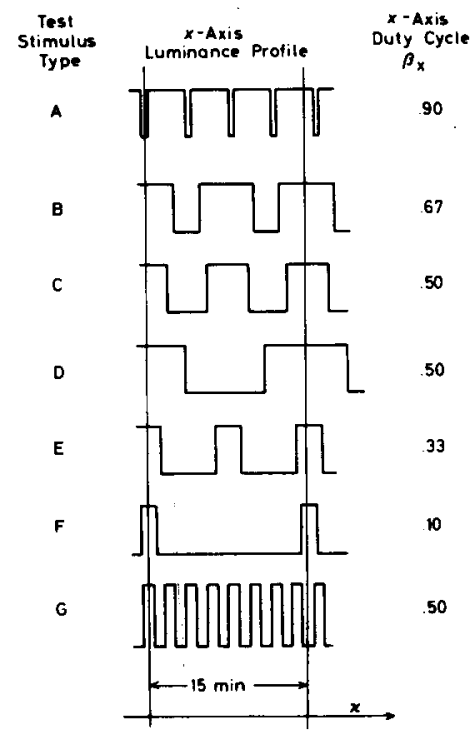

Figure 3. x-axis luminance profiles for the seven types of test patterns. In all cases, the higher luminance value (vertical axis) was $L_{\max }\left(120 \mathrm{~cd} / \mathrm{m}^{2}\right)$, and the lower value reached by the profile was $\mathrm{L}_{\mathrm{x}-\min }\left(62.5 \mathrm{~cd} / \mathrm{m}^{2}\right)$, for all stimuli. 

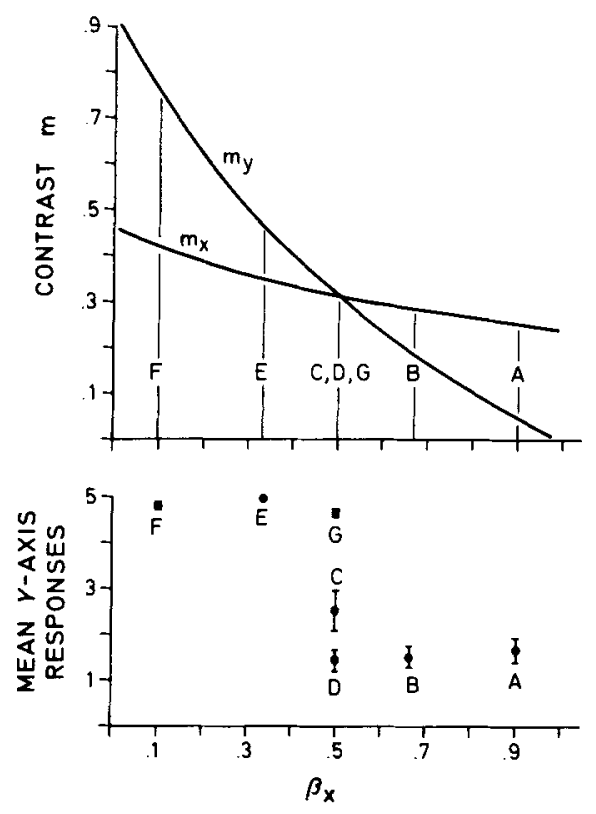

Figure 4. $x$-axis and $y$-axis contrast values for seven test patterns (upper panel), and mean number of $y$-axis responses for each pattern type (lower panel). The smooth curves in the upper panel show values of $x$-axis contrast $\left(m_{x}\right)$ and $y$-axis contrast $\left(m_{y}\right)$ that would occur if $\beta_{x}$ were varied continuously from .01 to .99 , under conditions described in the text. Vertical lines and letters locate $\beta_{x}$ and contrast values for stimuli actually used in the study. Note that stimuli were included with $m_{y}<m_{x}$ (types $A$ and $B$ ), $m_{y}=m_{x}$ (types $C, D$, and $G$ ), and $m_{y}>m_{x}$ (types $E$ and $F$ ). Points plotted in the lower half of the figure summarize results. Each point represents the mean number of $y$-axis responses for each pattern type. The points are shown over their $\beta_{x}$ values, so that each pattern's $x$ - and $y$-axis contrast values may be located easily in the upper panel.

\section{Procedure}

Only one subject participated in each experimental session. The session began with a 10-min adaptation phase in which the two appropriate adaptation stimuli were presented alternately at 10 sec intervals. The subject was instructed to move fixation around the "central area" of each stimulus.

Testing for the direction of McCollough aftereffects immediately followed the adaptation phase. During the test phase, each of the seven test stimulus types was presented five times; each type appeared at least once in each of the four possible orientations (the divided test pattern shown in Figure 1, for instance, could be presented as shown or in three other orientations). The 35 test slides were presented in a different order for each subject; orders were randomly determined with the constraint that every seven presentations include one appearance of each pattern type. During adaptation and testing, the room was dark except for light from the projector and screen. The $5^{\circ} \times 5^{\circ}$ test stimuli appeared in the middle of a large surrounding area of approximately $120 \mathrm{~cd} / \mathrm{m}^{2}$ produced by the projection beam.

We used a forced-choice procedure to assess color aftereffects elicited by these stimuli. Immediately before each test slide appeared, the subject was told how the two halves would be divided ("left and right" or "top and bottom"). The subject was instructed to inspect the pattern for at least "several" seconds and then, when ready, to write down on a prepared form which half appeared "redder." In the event that green but no red aftereffects appeared, the subject was told to indicate the half appearing "leasi green." After the experimental session, each subject was invited to write a description of the effects he or she had observed, and then wh dincuss these reports.

\section{RESULTS}

The forced choice responses for all subjects are summarized in the lower half of Figure 4. These responses were scored as being toward the y-axis if the subject designated as redder (or, equivalently, less green) the half of the test pattern in which the large 2-cpd bars, or "stripes," had the same orientation as the green 2-cpd pattern viewed during adaptation. This figure also shows the mean and standard error of $y$. axis response frequencies. The means for each pattern type are shown over their corresponding $\beta_{x}$ values, so that each pattern's respective contrast values $\left(m_{x}\right.$ and $\left.m_{y}\right)$ may be located easily in the upper panel. On the basis of these data, we conclude that $x$-axis responses predominate with $A, B$, and $D$ stimuli, and that $y$-axis responses predominate with $E, F$, and $\mathrm{G}$ stimuli.

The Friedman analysis of variance by ranks (Siegel, 1956) was applied to these data by rank ordering the seven test stimuli for each subject, according to the number of $y$-axis responses given for that pattern type. This analysis showed that $y$-axis response frequencies differed significantly among the seven stimulus types $\left(\chi^{2}=41.7, \mathrm{df}=6, \mathrm{p}<.001\right)$.

\section{DISCUSSION}

The major conclusions we draw from these results are: (1) The direction of McCollough effect responses to such stimuli can be predicted easily from pattern contrasts and a consideration of human contrast sensitivity characteristics: (2) responses cannot be predicted from similarities between adaptation and test pattern Fourier characteristics: and (3) pattern con-

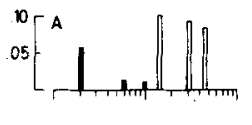

$X$-AXIS COMPONENTS 0 $\checkmark$-AXIS COMPONENTS I

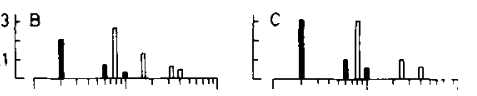

岁
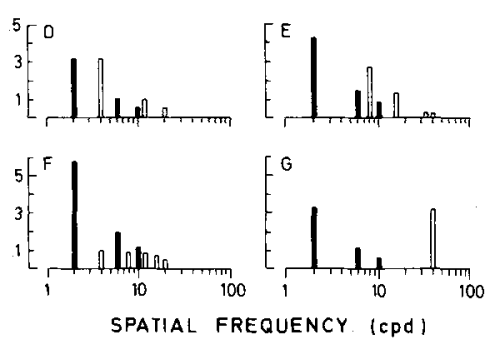

Figure 5. Frequency spectra for stimuli used in this study, showing Fourier characteristics along $x$ - and $y$-axis profiles (treating the profiles as infinite waveforms). Components greater than the 5th harmonic, phase relations, and components at $60-\mathrm{cpd}$ or higher frequencies are not shown. Fourier components of the adaptation patterns, also not shown, would appear at the same frequencies as all $y$-axis components represented by the dark bars $(2,6$, and $10 \mathrm{cpd})$. The vertical (amplitude) axis of this figure has the same scale for all pattern types except type $A$. 
trast, rather than black element width per se, determines the dominant direction of aftereffects. We discuss each of these conclusions separately.

In evaluating our results, we describe aftereffects as being "toward" one profile or the other. It will be useful here to clarify what this means. If a subject had adapted to, say, vertical green and black bars, we would expect subsequently viewed vertically oriented patterns to elicit a red McCollough aftereffect. A horizontal luminance profile defines a vertically oriented pattern. In each test pattern, the horizontal $\mathrm{x}$-axis was in one half and the horizontal $\mathrm{y}$-axis was in the other half. In this case, the appearance of a red aftereffect in a test pattern half with a horizontal $y$-axis would be called a response in the $y$ direction, or toward the y-axis. Within each test pattern half, an $\mathrm{x}$-axis and a perpendicular $\mathrm{y}$-axis were in competition for different aftereffect colors. We interpret the appearance of a y direction response as evidence that the $y$-axis profiles produced a stronger aftereffect than the $\mathrm{x}$-axis profiles.

\section{Contrast}

Figure 4 shows that when $m_{y}$ was greater than $\mathrm{m}_{\mathrm{x}}$ ( $\mathrm{E}$ and $\mathrm{F}$ stimuli), y direction responses predominated, and $x$ direction responses were dominant when $m_{y}$ was less than $m_{x}$ (A and $B$ stimuli). Thus, when contrasts of perpendicular profiles differed, McCollough effects tended toward the profile having greater contrast.

Consider the patterns having equal contrast in $x$ and $y$ dimensions, types $C, D$, and $G$. Each of these types had $\mathrm{m}_{\mathrm{x}}=\mathrm{m}_{\mathrm{y}}=.32$, and $\overline{\mathrm{L}}=91.3 \mathrm{~cd} / \mathrm{m}^{2}$; they also had equal amplitude (but different frequency) Fourier components in $\mathrm{x}$ and $\mathrm{y}$ dimensions (see Figure 5). However, these pattern types produced quite different distributions of $\mathrm{x}$ and $\mathrm{y}$ direction responses: mean $\mathrm{y}$-axis response frequencies to types $\mathrm{C}, \mathrm{D}$, and $G$, were, respectively, $2.5,1.5$, and 4.8 per subject. For these stimuli only, the Friedman ANOVA by ranks was applied to responses in the same manner as it was applied earlier for all responses. This analysis showed that these y-axis response frequencies also differed significantly among pattern types $\chi^{2}=14.4$, $\mathrm{df}=2, \mathrm{p}<.001$ ). We account for these differences by observing that when $m_{x}=m_{y}$, aftereffects tended toward the profile whose fundamental spatial frequency was associated with the greater human contrast sensitivity.

Examination of this issue requires an estimate of contrast sensitivity at different spatial frequencies. Assessment of contrast sensitivity at suprathreshold contrast levels is troublesome, but specification of sensitivity in terms of contrast thresholds has been reliably and abundantly documented. We have therefore arbitrarily chosen to base our discussion on a contrast sensitivity (threshold) function presented by Blakemore and Campbell (1969), although almost any other similarly obtained function will lead to identical conclusions. Their contrast thresholds were obtained at a mean luminance of $100 \mathrm{~cd} / \mathrm{m}^{2}$; our $C, D$, and $G$ stimuli had $\bar{L}=91.3 \mathrm{~cd} / \mathrm{m}^{2}$. We discuss only fundamental frequency components here, but the same conclusions follow if higher harmonic components are also considered.

Pattern type $C$ had a 2-cpd y-axis profile perpendicular to an 8-cpd $x$-axis profile (see Figure 5). Blakemore and Campbell show these to have about equal contrast thresholds. Our subjects produced a mean of $2.5 \mathrm{y}$-axis responses to these patterns, indicating no preference for responses in either direction. Blakemore and Campbell report greater sensitivity at $4 \mathrm{cpd}$ than at $2 \mathrm{cpd}$; our subjects produced a mean of $1.4 \mathrm{y}$-axis responses to type $\mathrm{D}$ stimuli, where a 2-cpd y-axis profile was in competition with a 4-cpd $x$-axis profile, thus showing a clear preference for responses toward the 4-cpd $x$-axis. Furthermore, Blakemore and Campbell report contrast thresholds about 100 times higher for 30 -cpd sinusoids than for 2 -cpd sinusoids. For pattern type $\mathrm{G}$, a mean of 4.8 $y$-axis responses per subject shows a very strong dominance of the 2-cpd y-axis profile over the 30 cpd $x$-axis profile. Thus, to the extent that inferences about suprathreshold contrast sensitivity can be made from threshold sensitivity, our results show that aftereffect direction is predicted by pattern contrast.

A number of earlier McCollough effect studies suggest that luminance contrast is a crucial variable in establishing and eliciting McCollough aftereffects. Indirect evidence, for example, was provided by Sharpe and Tees (1978), who found that aftereffect strength (measured as magnitude estimates) was greater for continuous test grating bars than for "interrupted" test grating bars. More direct evidence was provided by Stromeyer and Dawson (1978), who found that McCollough effects may be made sensitive to the polarity of luminance contrast differences, and that these effects do not appear when adaptation patterns have chromatic contrast only. From these studies and our own, we conclude that pattern contrast is a crucial factor in determining the direction of McCollough aftereffects.

\section{Fourier Characteristics}

Campbell and Robson (1968) hypothesized that the visual system contains channels selectively tuned to narrow bands of spatial frequencies. The hypothesis is attractive, for if one assumes independence among channels, and that their output magnitudes are approximately a linear function of input magnitudes, then methods of linear systems analysis may be applied to predict psychophysical responses from Fourier characteristics of visual stimuli. Since the Campbell and Robson hypothesis suggests that McCollougheffect responses should be predictable from this relation, we examine here the relationship between Fourier characteristics of our adaptation and test patterns. 
Referring again to Figure 5, consider the $\mathrm{x}$ - and $\mathrm{y}$ axis Fourier characteristics of stimulus type A. After adaptation to a 2-cpd squarewave, our subjects reported aftereffects primarily in the direction of a luminance profile with a 15-cpd fundamental ( $x$ axis) rather than toward a profile with a 2 -cpd fundamental (y-axis). This might have occurred because the 15-cpd $x$-axis fundamental had a larger amplitude (.098) than the perpendicular 2-cpd y-axis fundamental (.064). However, if one assumes that the McCollough effect results from sensitivity changes induced by adaptation stimuli, our results are difficult to account for strictly in terms of the Campbell and Robson hypothesis. It does not seem likely that adaptation to a 2-cpd squarewave should change sensitivity more in a 15 -cpd channel than it changes sensitivity in a 2-cpd channel.

Consider also test pattern type D, which elicited the lowest mean number of $y$-axis responses. Frequency components for both dimensions had equal amplitudes, and contrast was also equal along both axes. Complementary color aftereffects, however, were more often directed toward the set of frequencies different from adaptation frequencies (the 4-cpd $x$ axis) than toward the profile having the same frequencies (the 2-cpd y-axis profile). Pattern type $C$ also had equal amplitude components and equal contrast in both directions; here, a 2-cpd y-axis profile was in competition with an 8-cpd $x$-axis profile. Again, if aftereffects derive from sensitivity changes in narrow-band channels, one would expect our adaptation gratings to change sensitivity of the 2-cpd channel more than they change sensitivity of the 8-cpd channel, leading to a clear preference for y-axis responses. But, in this case, subjects produced their most mixed set of responses to type $C$ patterns (a mean of $2.5 \mathrm{y}$-axis responses out of five trials).

By contrast, test pattern types $\mathrm{E}, \mathrm{F}$, and $\mathrm{G}$, produced a majority of $y$-axis responses. For these three stimuli, aftereffects were almost always directed toward the profile with the same frequencies as adaptation grating profiles, that is, the y-axis. Thus, considering all seven test patterns, we find no discernible relation between aftereffects and similarity between adaptation and test-pattern Fourier characteristics. While many other investigators have argued that McCollough effects do depend on the relation between test pattern and adaptation pattern spatial frequencies (e.g., Sharpe \& Tees, 1978), we emphasize that human contrast sensitivity varies with spatial frequency, and the effects of sensitivity should be accounted for first before properties of aftereffects are attributed to their spatial frequency components.

\section{Black Element Width}

In this section, we examine the possibility that differences in widths of black test-pattern elements better predict aftereffect direction than do the contrast values considered earlier. Uhlarik and Osgood (1974) examined separately the effects of black-bar width and "slit" (white bar) width on the magnitude of orientation-contingent McCollough effects. They interpreted their results as showing that (1) test-pattern bar width associated with the strongest aftereffects increased as adaptation bar width increased, and (2) test-pattern slit width associated with the strongest aftereffects was independent of adapatationpattern slit width. They concluded that bar width was the critical variable influencing aftereffect strength. From their report, however, it is not possible to evaluate the effects of pattern contrast, which also varied among their test and adaptation stimuli.

In the present study, all test patterns had the same dark bar width in the $y$ dimension $(Y-y)$, but different dark element widths in the $x$ dimension $(X-$ $x)$ (see Figures 2 and 3 ). If aftereffect strength varies with dark element width, we should expect our wider $\mathrm{x}$-axis elements to produce more $\mathrm{x}$-direction responses than are produced by narrower $x$-axis elements.

Our analysis of the effects of element width is based on three rank orderings of the test stimuli. As Figure 3 shows, type $F$ patterns had the widest $x-$ axis dark elements, followed, in decreasing order of element widths, by patterns D, E, C, B, G, and A. Pattern type $D$ elicited the highest frequency of $x$ direction responses, followed, in decreasing order of $x$ direction responses, by patterns $B, A, C, G, F$, and $E$ (see the lower panel of Figure 4). To separate the effects of contrast differences from element width differences, we also rank ordered the stimuli in terms of $m_{x} / m_{y}$ values: pattern $A$ had the greatest ratio of $\mathrm{x}$-axis to $\mathrm{y}$-axis contrast, followed, in decreasing order of contrast ratios, by patterns, $B, C=D=G$, $E$, and $F$ (see the upper panel of Figure 4). Kandall $\tau$ partial correlation coefficients were then determined for these rank orders (Siegel, 1956). The partial correlation $\tau$ between element widths and $\mathrm{x}$-axis response frequencies, with the effects of $m_{x} / m_{y}$ held constant, was .000 . The partial correlation between $\mathrm{m}_{\mathrm{x}} / \mathrm{m}_{\mathrm{y}}$ ranks and $x$-axis response frequencies, with the effects of element width held constant, was .341 . Had we rank ordered stimuli according to human contrast sensitivity at profile fundamental frequencies, instead of by $m_{x} / m_{y}$ values, the difference between these $T$ values would have been substantially greater. We conclude that contrast rather than black element width is the crucial variable in determining the direction of McCollough aftereffects.

\section{Final Comments}

It might be argued that the effects we report are due to subjects' criteria for making responses, stra- 
tegies for scanning the test patterns, or, perhaps, the particular length of viewing time for each pattern. While space considerations do not permit detailed discussion of such possible alternate accounts, we found, in our own viewing of the test patterns, the same color effects described by the majority of subjects, and noted that these effects do not change with scanning direction or how attention is focused on particular parts of the pattern. We have also obtained the same results many times under less formal conditions with many professional associates. In addition, most subjects, in their verbal reports made after testing, indicated that color aftereffects were very stable, after two or three seconds of initial "fluctuation." It is possible that shorter test pattern inspection periods might produce different responses than those we obtained, but we do not believe our results would change with longer inspection periods.

\section{REFERENCES}

Blakemore. C., \& Campbell, F. W. On the existence of neurones in the human visual system selectively sensitive to the orientation and size of retinal images. Joumal of Physiology (London), 1969, 203, 237-260.

Campbell. F. W., \& Robson, J. G. Application of Fourier analysis to the visibility of gratings. Journal of Physiology (London), 1968, 197, 551-566.

Hoekstra, J., van der Goot, D. P. J., van den Brink, G., \& BILSEN, F. A. The influence of the number of cycles upon the contrast threshold for spatial sine wave patterns. Vision Research, 1974, 14, 365-368.

McCollough, C. Color adaptation of edge-detectors in the human visual system. Science, 1965, 149, 1115-1116.

Sharpe, L. T., \& Tees, R. C. Contour specificity of the McCollough effect. Perception \& Psychophysics, 1978, 23, 451.458

SIEGEL, S. Nonparametric statistics for the behavioral sciences. New York: McGraw-Hill, 1956.

Skowbo, D., Timney, B. N., Gentry, T. A., \& Morant, R. B. McCollough effects: Experimental findings and theoretical accounts. Psychological Bulletin, 1975, 82, 497.510.
Stromeyer, C. F., III, \& Dawson, B. M. Form-colour aftereffects: Selectivity to local luminance contrast. Perception, 1978, 7. 407-415.

Uhlarik, J. J., \& Osgood, A. G. The role of some spatial parameters of gratings on the McCollough effect. Perception \& Psychophysics, 1974, 15, 524-528.

\section{NOTES}

1. Frequency spectra for periodic waveforms of infinite length have components only at the fundamental frequency and higher harmonics, as represented by the spectra graphed in Figure 5. Our adaptation and test pattern profiles consisted of 10 cycles (or 5 cycles for one $x$ and one $y$ profile in each test pattern). Strictly speaking, finite waveforms such as those used here have continuous spectra, with the largest amplitude components located around the fundamental frequency of the periodic part of the waveform. It might be argued that our analysis and discussion section should be concerned with bands of frequencies rather than with discrete frequencies. However, an analysis of our stimuli and data based on the continuous spectra leads to the same conclusions as the discrete spectra analysis presented. Moreover, evidence indicates that visual sensitivity changes very little, if at all, as the number of cycles viewed increases beyond 5 (see, for example, Hoekstra, van der Goot, van den Brink, \& Bilsen, 1974). For these reasons, we present the simpler, more easily understood discrete spectra.

2. The more familiar index of contrast is a peak-to-peak contrast, $C$, often called Michelson contrast: $C=\left(L_{\max }-L_{\min }\right)$ / $\left(L_{\max }+L_{\min }\right)$. If $C$ values are computed for our stimuli, rather than the $m$ values shown in Figure 4 , contrast $C_{x}$ for all $x$-axis profiles is a constant .32 and $y$-axis contrast is a linear function of $\beta_{x}$. A graph of such values is similar to the upper panel in Figure 4, except that $C_{x}$ and $C_{y}$ functions appear as straight lines. The important point is that the ordinal relation between $x$ - and $y$-axis contrasts is the same for each pattern, regardless of which contrast ( $m$ or $C$ ) is computed. That is, for patterns $A$ and $B, C_{y}<C_{x}$; for patterns $C, D$, and $G, C_{y}=C_{x}$; and, for patterns $E$ and $F$, $C_{y}>C_{x}$. We chose to present $m$ rather than $C$ values because $\mathrm{m}$ reflects mean luminance levels of the stimuli.

(Received for publication November 27, 1978; revision accepted January $12,1979$. 